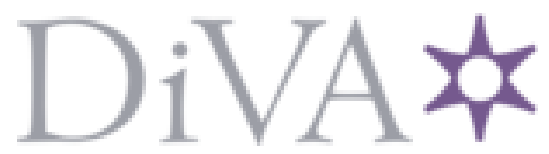

http://www.diva-portal.org

\title{
Preprint
}

This is the submitted version of a paper presented at International Conference on Probabilistic Methods Applied to Power Systems (PMAPS), 7-10 July 2014,Durham.

Citation for the original published paper:

Vardanyan, Y., Hesamzadeh, M. (2014)

Optimal bidding of a profit-maximizing hydropowerproducer in day-ahead and real-time markets.

In: Probabilistic Methods Applied to Power Systems (PMAPS), 2014 International Conference on (pp. 1-6). IEEE conference proceedings

http://dx.doi.org/10.1 109/PMAPS.2014.6960589

N.B. When citing this work, cite the original published paper.

Permanent link to this version:

http://urn.kb.se/resolve?urn=urn:nbn:se:kth:diva-165032 


\section{Optimal bidding of a profit-maximizing hydropower producer in day-ahead and real-time markets}

\author{
Yelena Vardanyan \\ Electricity Market Research Group \\ KTH Royal Institute of Technology \\ Email: yelena.vardanyan@ee.kth.se
}

\author{
Mohammad Hesamzadeh \\ Electricity Market Research Group \\ KTH Royal Institute of Technology \\ Email: mrhesamzadeh@ee.kth.se
}

\begin{abstract}
This paper develops a price-driven optimal bidding strategy to day-ahead and real-time markets for a profit maximizer hydro power producer. The electricity prices in different market places are unknown when the bidding takes place. The optimal bidding problem is modeled as a multi-stage stochastic program considering the market prices continuously clearing nature. Specifically for that purpose rolling planning is applied, which allow re-forecasting and re-dispatching according to the arrival of new information. The results have shown that, there is a value for hydropower producer to participate in real-time market.
\end{abstract}

\section{INTRODUCTION}

Within the past twenty years significant efforts were made throughout the world to deregulate electricity markets. Electricity is now a commodity which can be sold and purchased at the market with the price defined by demand and supply for each hour [1].

One advantage with the hydropower is the possibility to store energy, which allows the hydropower producer to act according to the market prices. This means, the hydropower producer has an opportunity to dispatch and sell power when the prices are high and store water for the future usage while the prices are not so attractive [2]. Therefore the optimal bidding strategy is very relevant to hydropower producers.

This paper develops multi-stage stochastic bidding strategy in day-ahead and real-time markets for a profit-maximizing hydropower producer. Special attention is given to model characteristics of real-time market prices applying rolling planning. Uncertain variables are forecasted using stochastic processes. Precisely, Mean Reverting and Jump Diffusion (MRJD) processes are applied hoping it can capture future electricity market prices. Markov model is used to predict the discrete behavior of real-time markets.

The paper is structured in the following way. Section II reviews the existing literature in the field. Section III explains methods to forecast electricity market prices and generate scenarios based on that. The multi-stage stochastic bidding model is developed in section IV. The model results are discussed and analysed in section V. Eventually, section VI concludes the paper.

\section{LITERATURE REVIEW}

Massive amount of work has been done in the field of short-term hydropower planning under uncertainty. Different type of models aiming to generate optimal bidding strategy (for different market places and under different conditions) for a price taker hydro power producer are available in the literature. A bidding strategy to day-ahead market for a price taker hydropower producer under uncertainty of the day-ahead market prices is developed in [3]. The author in [4] introduces multi-stage stochastic model under uncertainty of the spot market prices and water inflow. The first stage considers one-day production plan, while the other stages relates to the production of the following six days. Bidding strategy to day-ahead market for price taker hydropower producer considering the possibility to trade on the intra-day market has been explained in [5]. In [6] and [7], the authors use stochastic dynamic programming to derive bidding curves since there is no trivial way to estimate marginal cost in terms of water opportunity cost. A stochastic programming model for bidding to two-hour-ahead market is introduced in [8]. More contributions in the field of optimal bidding strategy include [9], [10] and [11].

In [12], the authors consider a generator, which offers electric power to day-ahead market under the price uncertainty. Bidding problem faced by a thermal price-taker producer under the price uncertainty is addressed in [13].

Further stochastic hydropower planning models are cited in the [14], [15], [16]. A stochastic hydropower planning model, which takes into account uncertainty related to the realtime market prices has been developed by Olsson in [17]. In addition, Olsson in [18] has introduced optimal bidding model to day-ahead market considering real-time market; modeling both market prices as uncertain variables. However, none of those mentioned above stochastic models capture the real-time market characteristics using rolling planning.

\section{ELECTRICITY PRICE FORECASTING}

The deregulation of electricity markets made the forecasting process of electricity prices more challenging. According to [19], in deregulated markets, electricity price dynamics reflect special characteristics of the particular market under study. Therefore, to recognize the main economic drivers, it is important to study the peculiarities of the electricity price 
dynamics. The scope of this section is to describe and predict electricity price behavior in day-ahead and real-time markets.

A huge number of studies have been curried out in the literature to model day-ahead market prices. Detailed review on the topic is given in [20]. Day-ahead market price forecasting techniques based on the time series models can be categorised in three groups: regression or causal models [21], [22], artificial intelligence based models [23] and stochastic processes [24]. In contrast, existing forecasting models for real-time market prices are few [25], [26].

In this work stochastic processes, namely Mean Reversion Jump Diffusion process (MRJD), are used to model day-ahead market prices. The success of those models is related to their ability to address the main features of electricity prices: mean reversion and spikes. To model real-time market prices the combination of MRJD stochastic process and Markov chain is used. Markov chain model addresses the discrete behavior of the prices and MRJD stochastic process addresses the continuous behavior of the prices [26].

\section{A. Mean Reversion Jump Diffusion process}

In general, a variable, which evolves over time in a way that is at least in part random refers as a stochastic process [27], [19]. For example, day-ahead market prices is stochastic, since its variation over time is predictable; increases during morning hours, decreases during day time, increases again during evening hours and decreases during the night. However, it is partly uncertain and unpredictable. Moreover, the price of day-ahead market is continuous-time stochastic process even if we measure the price at some fixed points in time.

A MRJD stochastic continuous-time process is defined in (1), where differential equation reflects the dynamics of the prices.

$$
d X_{t}=\alpha\left(\mu-X_{t}\right) d t+\sigma d W_{t}+J_{t} d P_{t},
$$

In (1) $\mu$ is the long term mean (also called the periodic attractor) and $\alpha$ is the speed of reversion. $W_{t}$ is Brownian motion (also known as Wiener process), which is responsible for frequent small fluctuations around $\mu$ proportional to $\sigma$. While Poisson process $P_{t}$ is producing non-frequent big spikes of size $J_{t}$ with $\lambda$ frequency of appearance for spikes.

To estimate the model parameters, the approach described in [28] is used. The continuous model is approximated by a discrete one by discretizing the price dynamics: $d t \longrightarrow \Delta t$, and in this work $\Delta t=1$ hour. It is assumed that the probability that more than one jump will occur during $\Delta t$ is nearly zero. Furthermore, the probability that one jump will happen is equal $\lambda \Delta t$. Alternatively, the probability that no jump will occur within $\Delta t$ small interval is equal $1-\lambda \Delta t$. The jump amplitude is normally distributed with the $\delta$ mean and $\gamma$ variance. Conditional of a jump occurrence during a given time interval the density function can be expressed $\sim N\left(0, \sigma^{2}\right)$ or $N\left(\delta, \sigma^{2}+\gamma^{2}\right)$. Thus, the model parameters can be estimated using Maximum Likelihood method, defining the likelihood function as a product of two densities (with and without a jump) weighted by the corresponding probabilities [29].

\section{B. Markov model}

To have a Markov model to describe the discrete behavior of the system for next 24 hours, first the probabilities of the transition matrix has to be estimated. Those parameters can be estimated using historical market prices. Based on the price series, $\left(b_{t}^{u p}, b_{t}^{\text {down }}\right)$ pairs are obtained, which show the state of the real-time market prices at time $t . b_{t}$ is a binary variable, which has the following form:

$$
b_{t}= \begin{cases}1 & \text { if real-time price is defined } \\ 0 & \text { if real-time price is not defined }\end{cases}
$$

Let $o_{t}$ be the variable which shows the price state at time $t$ as it is demonstrated below

$$
o_{t}=\left\{\begin{array}{ll}
1, & \text { if } \quad\left(b_{t}^{\text {up }}, b_{t}^{\text {down }}\right)=(0,0) \\
2, & \text { if } \quad\left(b_{t}^{\text {up }}, b_{t}^{\text {down }}\right)=(0,1) \\
3, & \text { if } \quad\left(b_{t}^{\text {up }}, b_{t}^{\text {down }}\right)=(1,0) \\
4, & \text { if } \quad\left(b_{t}^{\text {up }}, b_{t}^{\text {down }}\right)=(1,1)
\end{array} \quad t=1,2 \ldots T\right.
$$

Let $O_{i j}^{h}$ be a set defined in the following way

$$
O_{i j}^{h}=o_{t}: o_{t}=j, o_{t-1}=i, \ldots, o_{t-h}=i, t=h+1, \ldots, T
$$

then transition probabilities for $i, j=1, \ldots, 4$ can be calculated using transition (5)

$$
p_{i j}^{h}=\frac{\# O_{i j}^{h}}{\sum_{n=1}^{4} \# O_{i, n}^{h}}, \quad i, j=1, \ldots 4
$$

Having probability matrix estimated, it is possible to predict different scenarios for the state of the system for coming 24 hours.

\section{MAThematicAl MODEL}

When the day-ahead market is cleared, day-ahead market prices for all hours of the next day are set at once. Then, so called second level uncertainty related to the development of the real-time market takes place. Rules for submitting bids to real-time market are different from the one for the day-ahead market. The real-time market for a specific hour is closed very short before the delivery. Hence, the real-time market prices are settled according to the time progress. As a producer of a flexible power source, a hydropower producer has to make optimal decision (to save the water for the real-time market, or sell the whole electric power on the day-ahead market) under the uncertain data. Multistage stochastic programming technique is applicable in this context. 


\section{A. Rolling planning}

The intermittent wind power impact on the power system, which is more volatile prices in day-ahead and real-time markets, is considered by constructing a scenario tree. The scenario tree keeps information about the forecasted prices in different market places with different forecast horizon, corresponding to each hour in the optimization period. The scenarios in the scenario tree are represented as different outcomes of the prices accompanied with corresponding probabilities.

As it was explained earlier the rules for day-ahead market are different that of real-time market. The information about real-time market is revealed continuously. Hence, in order to benefit from the information released over time, instead of simulating the whole planning period by only one scenario tree, the model is formulated using rolling planning by introducing multi-stage recursion.

In the multi-stage stochastic problems the decision variables are categorized as here and now (also in the literature known as root) decisions and wait and see (also known as recourse) decisions [30]. Root decisions have to be taken before the uncertainties are revealed, while recourse decisions can be taken after the outcomes of the uncertain events are released. Thus, correcting actions can be done using recourse decisions. For example, hydro power producer has to decide bid volumes (root decision) to the day-ahead market under uncertainties. The bids in many markets have to be put 12-36 hours in advance. Because of the wind power share in the power system, electricity prices in the different market places are volatile. Therefore, some recourse actions might be needed in the form of up or down regulation in the real-time market.

As new information is arriving in the hourly bases, the idealistic case would be to update the evolved information in the planning model in the hourly bases. However, the size of the stochastic models increase exponentially over time. Thus, to keep the developed model computationally tractable the information is updated in the developed model in every six hours; the developed model steps forward in time with six hours step using rolling planning. For each time step new scenario tree is used, which contains the updated forecasts for real-time market prices. Hence, for each time step the developed problem is solved (from now on we will call iteration) with the updated scenario tree.

For each iteration two-stage stochastic problem is solved. After the first iteration the volume sold to day-ahead market for each hour is known. In the consecutive iterations the variable corresponding to the bid amount to day-ahead market is fixed to the values found in the first iteration, in a way that the dispatch to day-ahead market is respected when optimal trading of real-time market takes place. In addition, after the first iteration bids for real-time market for the first six hours are fixed too.

\section{B. Short term hydropower planning}

The objective function of the optimization problem applicable for power plants located in the same river volley is presented below.

$$
\begin{array}{r}
\text { Max } \sum_{s} P^{s}\left[\sum_{t}\left(\lambda_{s, t} y_{s, t}+\lambda_{s, t}^{u p} d_{s, t}^{u p}-\lambda_{s, t}^{\text {down }} d_{s, t}^{\text {down}}\right)\right. \\
\left.+\lambda_{f} \sum_{j} \sum_{r \in R_{j}} \gamma_{r} m_{s, j}^{T}\right]
\end{array}
$$

The objective function (6) consists of expected profit from the trading on day-ahead market, real-time market and future trading, where $s$ is index for scenarios associated with $P^{s}$ probabilities respectively. $\lambda_{s, t}$ are the prices on day-ahead market, $\lambda_{s, t}^{u p}$ and $\lambda_{s, t}^{\text {down }}$ are prices on real-time market related to up and down regulation correspondingly and finally $\lambda_{f}$ is water opportunity cost. $y_{s, t}$ is dispatch level to day-ahead market, $d_{s, t}^{u p}$ and $d_{s, t}^{\text {down }}$ are dispatch amount to real-time market. $m_{s, j}^{T}$ is the end content of the reservoirs and $\gamma$ is expected future production equivalent for a plant.

The third term in (6) has negative sign, since the hydropower producer is buying energy from TSO, instead of producing it. For a hydropower producer the incentive is to increase the profit; buying water when the prices are low having an intention to sell it with more favorable prices.

The first constraint is day-ahead market bidding rule. In order to model the bidding process, the possible bidding prices are fixed: equidistance price points are selected and the corresponding bid volumes are considered as variables (similar to the approach in [3]). Let $i$ be the index for the possible bid prices and $\rho_{i}$ represents these prices. Then, a fundamental rule is applied to couple bid volumes $x_{i, t}$ and dispatched volumes $y_{t}$ : for each hour if $\rho_{i} \leq \lambda_{t} \leq \rho_{i+1}$ then $y_{t}=\sum_{p=0}^{i} x_{i-p, t}$, where $p=1,2 \ldots i$.

$$
y_{s, t}=\sum_{p=0}^{i} x_{i-p, t} \quad \text { if } \quad \rho_{i} \leq \lambda_{t}^{s} \leq \rho_{i+1},
$$

Constraint (7) states the bidding rule to day-ahead market considering uncertainties.

$$
\begin{gathered}
d_{s, t}^{u p}=\sum_{p=0}^{i} z_{i-p, t}^{u p} \quad \text { if } \quad \rho_{i} \leq \lambda_{s, t}^{u p} \leq \rho_{i+1}, \\
d_{s, t}^{\text {down }}=\sum_{p=0}^{i} z_{i-p, t}^{\text {down }} \quad \text { if } \quad \rho_{i} \leq \lambda_{s, t}^{\text {down }} \leq \rho_{i+1},
\end{gathered}
$$

In the same way (8) and (9) are stating the bidding rules in real-time market for up and down regulation correspondingly. Here $z_{i, t}^{u p}$ and $z_{i, t}^{\text {down }}$ is the up and down regulation bid volume and $d_{s, t}^{u p}$ and $d_{s, t}^{\text {down }}$ is the dispatch volume for up/down regulation. Similarly, $\lambda_{s, t}^{u p}$ and $\lambda_{s, t}^{\text {down }}$ are the realized real-time market prices for up and down regulated bids respectively.

$$
\begin{array}{r}
m_{j, t}^{s}=m_{j, t-1}^{s}-\sum_{n} Q_{j, t, n}^{s}-S_{j, t}^{s}+\sum_{n} Q_{j-1, t-\tau_{j}, n}^{s} \\
+S_{j-1, t-\tau_{j}}^{s}+I_{j, t}, s \in S, t \in T,
\end{array}
$$

As it is necessary to keep the hydrological balance between reservoirs a constraint (10) is introduced: for each hour the reservoir's new contents $\left(m_{j, t}^{s}\right)$ are equal to its old contents 
$\left(m_{j, t-1}^{s}\right)$ plus water inflow $\left(I_{j, t}\right)$ minus water outflow. The discharge and the spillage $\left(S_{j-1, t-\tau_{j}}^{s}\right)$ from the upper reservoir flow in the reservoir located downstream with delay time $(\tau)$.

$$
G_{j, t}^{s} \leq \sum_{n} \mu_{j, n} Q_{j, t, n}^{s},
$$

In addition, constraint (11) bounds hourly generation for each operated power plant according to its maximum discharge capacity and marginal production equivalent. Here $G_{j . t}^{s}$ is generation level and $Q_{j, t, n}^{s}$ is discharged volume for hourly bids.

The quantity traded on the day-ahead and real-time markets should be equal to the total output of the generation units for each hour. This leads to the constraint (12).

$$
y_{s, t}+d_{s, t}^{u p}=d_{s, t}^{d o w n}+\sum_{j} G_{j, t}^{s}, s \in S, t \in T,
$$

Limitation on down regulated quantity is modelled by constraint (13): the down regulated quantity $d_{s, t}^{\text {down }}$ can not exceed the bid volume to day-ahead market for each hour.

$$
d_{s, t}^{d o w n} \leq y_{s, t}
$$

Last two constraints together put bounds on hourly discharge level and on hourly water storage level respectively.

$$
\begin{aligned}
Q_{j, t, n}^{s} & \leq \bar{q}_{j, n}, \\
m_{j, t}^{s} & \leq \bar{m}_{j},
\end{aligned}
$$

Moreover, all variables $\in R_{+}, \bar{q}_{j, n}$ and $\bar{m}_{j}$ are maximum discharge level and maximum reservoir content respectively.

The stochastic program is summarized below:

$$
\begin{array}{r}
\mathbf{M} a x \sum_{s} P^{s}\left[\sum_{t}\left(\lambda_{s, t} y_{s, t}+\lambda_{s, t}^{u p} d_{s, t}^{u p}-\lambda_{s, t}^{\text {down }} d_{s, t}^{\text {down }}\right)\right. \\
\left.+\lambda_{f} \sum_{j} \sum_{r \in R_{j}} \gamma_{r} m_{s, j}^{T}\right]
\end{array}
$$

subject to $(7)-(15)$

\section{RESULTS AND DISCUSSIONS}

To test the model three cascaded reservoirs, located on the same river volley, are studied. The upper reservoir is larger, which is followed by two smaller reservoirs. Every reservoir has local inflow. In addition, each reservoir has a power station which contains a turbine, which by transferring the potential energy of the water to electrical energy generates electricity. Afterwards, the water flows downstream and is stored in the lower plant's reservoir, until released through the lower plants turbine. The data for the initial reservoirs content and the inflow level are according to Swedish hydropower plants. Table II summarizes the maximum storage capacity, the maximum design flow, the maximum production capacity for each power plants and water delay time [31]. For the dayahead and real-time price predictions, the historical data are taken from the Nord Pool website from 2012 March 10 to 2013 March 10.
The forecasting models described in section III are used to predict market prices and generate scenario tree which is used to represent day-ahead and real-time market prices in the optimization model presented in section IV. The optimization model is programmed in GAMS platform and CPLEX solver is used to solve it.

Table I

DATA FOR POWER PLANTS

\begin{tabular}{l|l|l|l|l}
\hline & $\bar{m}_{j}(\mathrm{HE})$ & $\bar{Q}_{j}\left(\mathrm{~m}^{3} / s\right)$ & $\bar{G}_{j}(\mathrm{MW})$ & $\tau_{j}(\mathrm{~h})$ \\
\hline I & 305856 & 340 & 95 & 0 \\
\hline II & 1392 & 310 & 50 & 0.5 \\
\hline III & 4008 & 330 & 90 & 2 \\
\hline
\end{tabular}

All assumptions made in the optimization model are listed below:

- The future electricity price is considered deterministic setting $50 € / M W h$ and is estimated taking the average value of futures and forward contracts.

- Initial reservoir content is considered $60 \%$ full.

- The hydropower producer is a price taker.

- Seven day-ahead market price scenarios are generated for each hour.

- For each day-ahead market price scenario three upward regulated and three downward regulating price scenarios are generated.

- Water delay time is considered.

- The possible fixed bidding prices are $35 € / M W h$, $50 € / M W h$ and $65 € / M W h$, which are estimated based on generated scenarios.

To show that 21 scenarios are capable to generate accurate and stabile solution, a stability test is curried out. For that purpose the model is run while increasing the number of the scenarios in the scenario tree. As one can see from the Fig. 1 the changes in objective function value are negligible from hours 17 to 21 . Hence, for this study, a scenario tree with 21 scenarios gives an acceptable approximation.

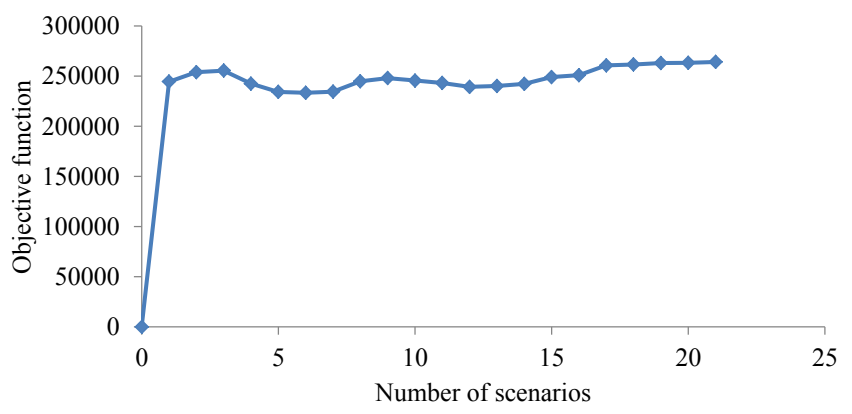

Figure 1. Changes in the objective function value vs. the number of scenarios considered.

Table V lists objective function optimal value for each iteration. The objective value is decreasing since after each iteration we are adding new constraints to the problem. This makes the feasible set tighter, accordingly the optimal value gets smaller through the iterations. 
Table II

OBJECTIVE FUNCTION VALUE

\begin{tabular}{l|l}
\hline Iterations & Opt. value (Euro) \\
\hline I & 265,526 \\
\hline II & 265,449 \\
\hline III & 264,880 \\
\hline IV & 264,116 \\
\hline
\end{tabular}

To show the working procedure when the problem is modeled with the rolling planning, up regulation bids are illustrated for all iterations in Fig. 2. After the first iteration (planning period) offered up regulation bids for the first six hours are fixed and the second planning takes place feeded by a new scenario tree, which considers new information revealed over time. From the Fig. 2 one can see that, after the first iteration, offered up regulated bids for the first six hours are remaining unchanged until the last iteration. However, offered bids for the remaining hours still can be changed depending on the updated information. For example, after the first iteration the offered bid volume for up regulation at hour seven is $33,15 M W h$, which changes and remains zero after the second iteration. Another change in offered volume for up regulation bid happens at hour 19 after the last iteration.

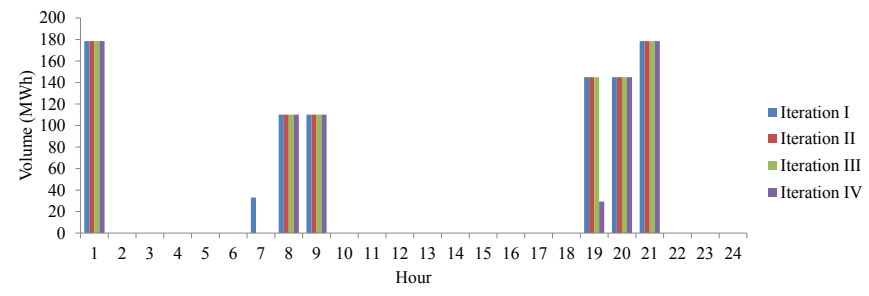

Figure 2. Up regulated bids for all iterations of a hydropower producer

Finally, the bidding volumes to different market places for each hour can be seen from Fig. 3. According to Fig.3 the

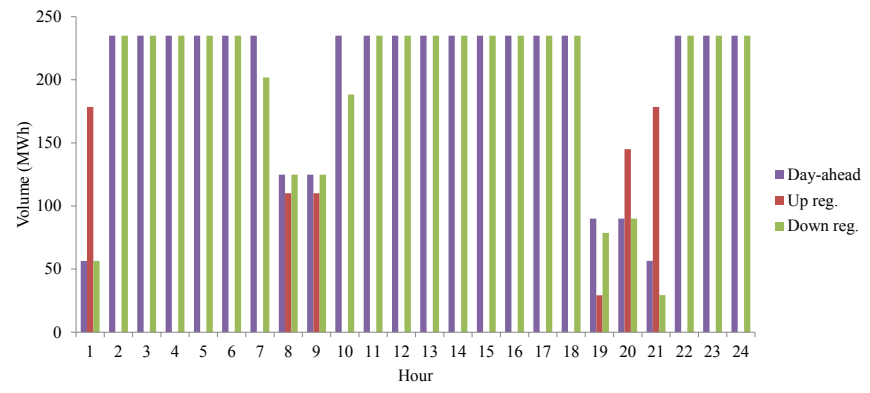

Figure 3. Bidding volumes to different market places of a hydropower producer

model suggests to bid to day-ahead market for almost every hour with the maximum capacity. However, the model then does correcting action by offering down regulation on realtime market. When the model bids to day-ahead market and put an offer to bring it back on real-time market, apparently the realized market price scenarios for up-regulation on real- time market are not attractive to offer some quantity. Thus, for those hours the model saves the water for the future usage.

The fact that, for most of the hours the model suggests to bid to day-ahead market and down regulated market with the maximum capacity is logical from the model point of view, since the problem is modeled for a profit maximizer hydropower producer. If the model expects some gain it will bid the maximum capacity aiming to maximize the profit. However, in real world applications some modification of the original problem might be needed [32], [33]. Moreover, the introduction of the variance of the profit in the objective function might consider the risk related to real-time market more thoroughly.

In addition, the model suggests up-regulation only at hours one, eight and nine in the morning and at hours from seven to nine in the evening. Apparently, those hours corresponds to the peak demand hours; hence the results are reasonable.

To validate the model results, the realised market prices of day-ahead and real-time markets for March 11, 2013 are analysed carefully (March 11, 2013 is the date for which the planning is done). The suggested periods for up-regulation by the model correspond to the real up-regulated periods. However, in reality up regulation continued until midnight starting from seven in the evening, while the model offered only up regulation bids at hours from seven to nine.

To estimate the hydropower producer's gain while participating to both day-ahead and real-time markets the results are compared with the model results, which only considers bidding to day-ahead market. The objective function value for the proposed model is $264,116 €$ (last row in Table V). And the objective function value for the benchmark model is $255,650 €$. Thus, the hydropower producer gains $8466 €$ participating also in real-time market.

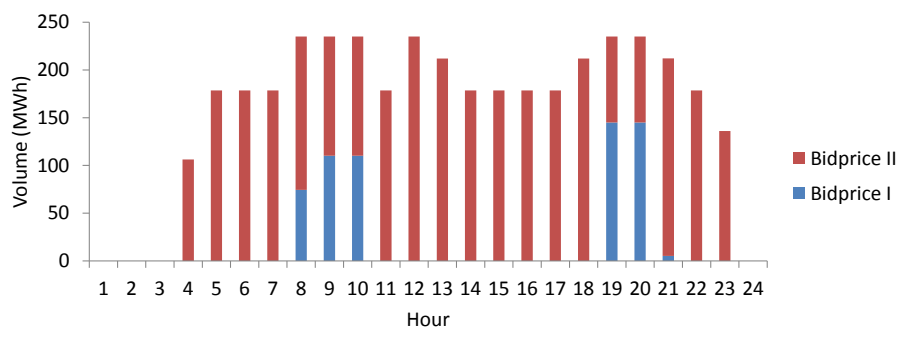

Figure 4. Bidding volumes of a hydropower producer to day-ahead market: Benchmark model

Another interesting point to notice is the bidding strategy of a hydropower producer to day-ahead market for both models. Day-ahead bids from the proposed model are illustrated in Fig. 5 and from the benchmark model are shown in Fig. 4. From Fig. 5, one can see that for nearly all hours, the hydropower producer bids to day-ahead market when the realized market prices are at least $35 € / M W h$. Apparently, here the hydropower producer counts on the correcting action on the real-time market via downward regulation. However, the benchmark model, which does not consider real-time market, bids only 


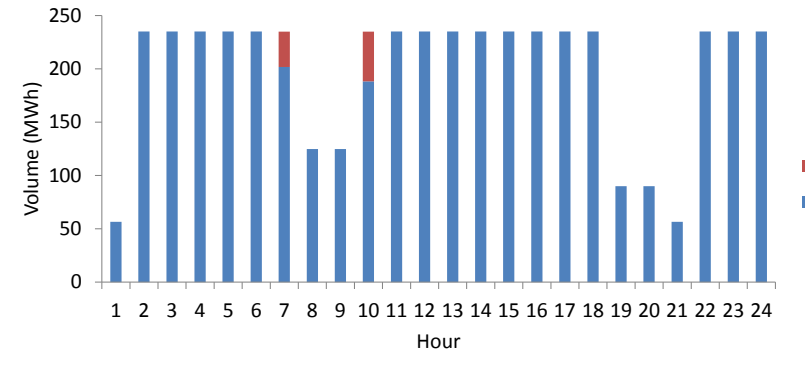

Figure 5. Bidding volumes of a hydropower producer to day-ahead market: Proposed model

when the realized market prices are higher than the water opportunity cost $(50 € / M W h)$ Fig. 4.

\section{CONCLUSION}

The optimal bidding decision is very relevant and meanwhile challenging issue for a profit-maximizing hydropower producer. This paper develops a model to calculate optimal bidding strategy of a hydropower producer. The proposed model generates bidding curves for two consecutive markets for each hour in the planning period. The developed model uses multi-stage stochastic programming tool to deal with the decision making process under uncertainty. Particularly, rolling planning is used to benefit from the continuously arriving information. In addition, in this work day-ahead and real-time market prices are modelled as MRJD stochastic processes.

The proposed stochastic model generates bidding curves for both day-ahead and real-time markets for each hour. Moreover, the results from our developed model are compared with the ones from a benchmark model where only day-ahead market is modelled. The comparison analyses show that there is a gain for hydropower producer to participate in both day-ahead and real-time market. The next step will be to reformulate our model considering the profit risk of a hydropower producer.

\section{REFERENCES}

[1] "The Swedish electricity market and the role of Svenska Kraftnät," tech. rep., Svenska Kraftnät, 2007.

[2] Y. Vardanyan, "On stochastic optimization for short-term hydropower planning," licentiate thesis, Electrical Systems, 2012.

[3] S.-E. Fleten and T. K. Kristoffersen, "Stochastic programming for optimizing bidding strategies of a Nordic hydropower producer," European Journal of Operation Research, vol. 181, 2007.

[4] S.-E. Fleten and T. K. Kristoffersen, "Short-term hydropower production planning by stochastic programming," Computers and Operations Research, vol. 35, 2008.

[5] E. Fariba and S.-E. Fleten, "Day-ahead market bidding for a nordic hydropower producer: taking the elbas market into account," Computational Management Science, vol. 8, pp. 75-101, 2011.

[6] G. Pritchard and G. Zakeri, "Market offering strategies for hydroelectric generators," Operations Research, vol. 51, 2003.

[7] G. Pritchard, A. B. Philpott, and P. Neame, "Hydroelectric reservoir optimization in a pool market," Mathematical Programming, vol. 103, 2005.

[8] D. De Ladurantaye, M. Gendreau, and J. Potvin, "Strategic bidding for price-taker hydroelectricity producer," IEEE Transactions on Power Systems, vol. 22, 2007.

[9] J. Contreras, O. Candiles, I. de la Fuente J., and T. Gomez, "A cobweb bidding model for competitive electricity markets," IEEE Transactions on Power Systems, vol. 17, 2002.
[10] N. Lu, J. Chow, and A. A. Desrochers, "Pumped-stirage hydro-turbine bidding strategies in a competitive electricity market," IEEE Transactions on Power Systems, vol. 19, 2004.

[11] E. Ni, P. Luh, and S. Rourke, "Optimal integrated generation bidding and scheduling with risk management under a deregulated market," IEEE Transactions on Power Systems, vol. 19, 2004.

[12] P. Neame, A. B. Philpott, and G. Pritchard, "Offer stack optimization in electricity markets," Operations Research, vol. 51, 2003.

[13] A. J. Conejo, F. J. Nogales, and J. M. Arroyo, "Price-taker bidding strategy under price uncertainty," in Proc. IEEE Power Engineering Society General Meeting, vol. 2, 2003.

[14] A. B. Philpott and H. Craddock, M. Waterer, "Theory and methodology: hydro-electric unit commitment subject to uncertain demand," European Jurnals of Operation Research, vol. 125, 2000.

[15] M. P. Nowak, R. Schultz, and M. Westphalen, "A stochastic integer programming model for incorporating day-ahead trading of electricity into hydro-thermal unit commitment," Optimization and Engineering, vol. 6, 2005.

[16] J. G. Gonzalez, R. M. R. Muela, L. M. Santos, and A. M. Gonzalez, "Stochastic joint optimization of wind generation and pumped-storage units in an electricity market," IEEE Transactions on Power Systems, vol. 23,2008

[17] M. Olsson and L. Söder, "Hydropower planning including trade-off between energy and reserve markets," in Proc. IEEE Bologna Power Tech, vol. 1, 2003.

[18] M. Olsson, On optimal hydropower bidding in systems with wind power. $\mathrm{PhD}$ thesis, Dept. of Electrical Engineering, KTH, 2009.

[19] S. Fiorenzani, Quantitative methods for electricity trading and risk management. Palgrave macmillan, 2006.

[20] K. S. Aggarwal, M. Saini, and A. Kumar, "Electricity price forecasting in deregulated markets: A review and evaluation," Electrical Power and Energy Systems, vol. 31, pp. 13-22, 2009.

[21] J. Contreras, R. Espinola, F. J. Nogales, and A. J. Conejo, "Arima models to predict next-day electricity prices," IEEE Transactions on Power Syatems, vol. 18, no. 3, 2003.

[22] R. C. Garcia, J. Contreras, M. van Akkeren, and J. B. C. Garcia, "A garch forecasting model to predict day-ahead electricity prices," IEEE Transactions on Power Syatems, vol. 20, no. 2, 2005.

[23] B. Szkuta, L. Sanabria, and T. Dillon, "Electricity price short-term forecasting using artificial neural networks," IEEE Transactions on Power Systems, vol. 14, 1999.

[24] R. Weron, M. Bierbrauer, and S. Truck, "Modeling electricity pricees: jump diffusion and regime sweitching," tech. rep., Hugo steinhous center for stochastic methods Wroclaw university of technology and chair of econimetrics and statistics, university of Karlsrune, 2001.

[25] K. Skytte, "The regulating power market on the Nordic power exchange Nord Pool: an econometric analysis," Energy Economics, vol. 21, 1999.

[26] M. Olsson and L. Söder, "Modeling real-time balancing power market prices using combined sarima and markov processes," IEEE Transactions on Power Systems, vol. 23, 2008.

[27] A. Dixit and R. Pindyck, Investment under uncertainty. Princeton University Press, 1994

[28] C. Ball and W. Torous, "A simplified jump process for common stock returnes," The journal of financial and quantitative analysis, vol. 18, 1983.

[29] R. Weron, "Marlab function to simulate, estimate and predict mean reverting jump diffusion processes," in Statistical software components M429004, Boston College Department of Economics, 2010.

[30] J. R. Birge and F. Louveaux, Introduction to stochastic programming. Springer-Verlag New York Inc., 1997.

[31] S. Angelin and etc., Hydro Power in Sweden. Svenska Kraftverksföreningen, 1987

[32] Y. Vardanyan, L. Söder, and M. Amelin, "Hydropower bidding strategies to day-ahead and real-time markets: different approaches," in 24th International Workshop on Database and Expert Systems Applications, 2013.

[33] Y. Vardanyan, M. Amelin, and M. Hesamzadeh, "Short-term hydropower planning with uncertain wind power production," in IEEE Power \& Energy Society General Meeting, 2013. 